\section{Clostridiodes difficile in COVID-19 Patients, Detroit, Michigan, USA, March-April 2020}

\author{
Avnish Sandhu, Glenn Tillotson, Jordan Polistico, \\ Hossein Salimnia, Mara Cranis, Judy Moshos, \\ Lori Cullen, Lavina Jabbo, Lawrence Diebel, \\ Teena Chopra
}

Author affiliations: Detroit Medical Center, Detroit, Michigan, USA (A. Sandhu, J. Polistico, H. Salimnia, M. Cranis, J. Moshos,

L. Cullen, L. Jabbo, T. Chopra); Wayne State University School of Medicine, Detroit (A. Sandhu, J. Polistico, H. Salimnia, L. Diebel, T. Chopra); GST Micro LLC, Henrico, Virginia, USA (G. Tillotson)

DOI: https://doi.org/10.3201/eid2609.202126

We describe 9 patients at a medical center in Detroit, Michigan, USA, with severe acute respiratory syndrome coronavirus 2 and Clostridiodes difficile. Both infections can manifest as digestive symptoms and merit screening when assessing patients with diarrhea during the coronavirus disease pandemic. These coinfections also highlight the continued importance of antimicrobial stewardship.

Coronavirus disease (COVID-19), which is caused by infection with severe acute respiratory syndrome coronavirus 2 (SARS-CoV-2), predominantly includes pulmonary symptoms; however, $<10 \%$ of cases also include gastrointestinal events, including abdominal pain, diarrhea, and vomiting (1-4). During the COVID-19 pandemic, clinicians must be vigilant of co-infections in patients with COVID-19.

Several studies have collected data on concomitant antibiotic use in patients with COVID-19. A single-center study of 52 critically ill patients cited hospital-acquired infection in only 7 (13.5\%) patients, yet $49(94 \%)$ patients received antibiotic therapy (5). Another study, which analyzed 113 deceased patients from a cohort of 799 moderate-to-severely ill COVID-19 patients during January 13-February 12, 2020, reported that 105 (93\%) deceased patients and $144(89 \%)$ survivors had received empiric antibacterial therapy with either moxifloxacin, cefoperazone, or azithromycin (6). These antibiotics are strongly associated with C. difficile infection (CDI) (7). We report an observation of $\mathrm{CDI}$ as a co-occurrence or sequalae of overuse of antibiotics in COVID-19 patients.

We conducted a clinical surveillance review of CDI for all laboratory-confirmed COVID-19 patients treated at any of the hospitals belonging to
Detroit Medical Center (Detroit, Michigan, USA). We screened patients by using TheraDoc software (https:/ / www.theradoc.com) during March 11-April 22,2020 . We abstracted data regarding baseline demographics, medical history, symptoms, laboratory values, microbiologic findings, concomitant antibiotic use, and treatment for CDI. We obtained institutional review board approval for this study.

We identified 9 cases of co-infection with SARSCoV-2 and C. difficile. This cohort mainly included elderly patients who were predominantly female (Table). The rate of CDI at the center was 3.32/10,000 patientdays during January-February 2020 and increased to 3.6/10,000 patient-days during March-April 2020.

We noted prior CDI in 3 patients; these infections occurred 1-4 months before admission. All patients were confirmed to be positive for $C$. difficile by PCR and showed symptoms of diarrhea in addition to other characteristic signs and symptoms, such as abdominal pain, nausea, and vomiting. Two patients had diarrhea and were found to be positive for $C$. difficile at admission, whereas the remaining 7 had onset of diarrhea only after COVID-19 diagnosis; median duration from CDI diagnosis to COVID-19 diagnosis in these 7 patients was 6 days. This group of patients

\begin{tabular}{|c|c|}
\hline \multicolumn{2}{|c|}{$\begin{array}{l}\text { Table. Baseline demographic and clinical characteristics of } 9 \\
\text { patients with Clostridium difficile and severe acute respiratory } \\
\text { syndrome coronavirus } 2 \text { co-infection, Detroit, Michigan, USA, } \\
\text { March-April } 2020^{*}\end{array}$} \\
\hline Characteristic & Value \\
\hline Age, $\mathrm{y}$, median & 75 \\
\hline \multicolumn{2}{|l|}{ Sex } \\
\hline $\mathrm{F}$ & 7 \\
\hline M & 2 \\
\hline \multicolumn{2}{|l|}{ Race } \\
\hline African American & 6 \\
\hline Caucasian & 1 \\
\hline Unknown & 2 \\
\hline Hospitalization in prior $60 \mathrm{~d}$ & 5 \\
\hline Required intensive care unit and vasopressors & 4 \\
\hline ATLAS score, median $\dagger$ & 6 \\
\hline Charlson comorbidity index score, median & 8 \\
\hline \multicolumn{2}{|l|}{ Symptoms at admission } \\
\hline Cough & 4 \\
\hline Shortness of breath & 3 \\
\hline Fever & 4 \\
\hline Diarrhea and abdominal pain & 2 \\
\hline \multicolumn{2}{|l|}{ Laboratory results } \\
\hline Ferritin, ng/mL, median $\ddagger$ & $1,459.4$ \\
\hline Leukocyte count, $\times 10^{3}$ cells $/ \mathrm{mm}^{3}$ ), average & 12.0 \\
\hline Creatinine, $\mathrm{mg} / \mathrm{dL}$, average $\S$ & 4.22 \\
\hline \multicolumn{2}{|l|}{ Microbiologic findings } \\
\hline Blood culture positive & 2 \\
\hline Respiratory culture positive & 2 \\
\hline \multicolumn{2}{|c|}{$\begin{array}{l}\text { *Values indicate no. patients unless otherwise indicated. Some patients } \\
\text { had }>1 \text { symptom. } \\
\text { †Scoring information available at https://www.mdcalc.com/atlas-score- } \\
\text { clostridium-difficile-infection. } \\
\text { †Ferritin was only obtained in } 8 \text { patients. } \\
\text { §Three patients were on dialysis. }\end{array}$} \\
\hline
\end{tabular}


were severely ill, having high ATLAS scores (https:/ / www.mdcalc.com/atlas-score-clostridium-difficileinfection) and multiple underlying conditions; hypertension $(n=8)$ and diabetes $(n=5)$ were the most frequent of these conditions.

Three patients received antibiotics in the month before admission; 8 received antibiotics at admission. One patient was initiated on antibiotics on day 15; this patient was also receiving antibiotics the month before admission. The most commonly administered antibiotics were cefepime $(n=5)$, ceftriaxone $(n=3)$, meropenem $(n=2)$, and azithromycin $(n=2)$. Specific CDI therapies were oral vancomycin $(n=6)$; vancomycin and intravenous metronidazole $(\mathrm{n}=1)$; no treatment $(\mathrm{n}=1)$; and a combination of oral vancomycin, intravenous metronidazole, rectal vancomycin, fidaxomicin, and fecal microbiota transplantation $(\mathrm{n}=1)$. One patient who did not receive antibiotics was considered to be colonized with $C$. difficile. Four (44.4\%) patients died during hospital admission, 1 (11.1\%) was discharged to hospice, $1(11.1 \%)$ is still hospitalized, and $3(33.3 \%)$ were discharged to a longterm care facility.

CDI is a challenging disease, with a recurrence rate of $15 \%-20 \%$ and a mortality rate of $5 \%(8)$. When CDI is present as a co-infection with COVID-19, CDI therapy can be difficult to monitor if diarrhea persists because of COVID-19.

These cases highlight the importance of judicious use of antibiotics for potential secondary bacterial infection in patients with COVID-19. Antibiotics are known to have unintended consequences, such as $C$. difficile infection. All 9 patients received antibiotics; the median duration of antibiotic use before PCRpositive CDI was 5 days. All patients in our cohort were elderly, an age group at higher risk for complications from overuse of antibiotics, such as adverse events, antibiotic resistance, and concomitant infections like CDI (9). Secondary infections on top of CDI can increase the risk for death in patients with severe COVID-19; in this cohort, 4 patients died and 1 was discharged to hospice. To prevent CDI co-infections during the COVID-19 pandemic, integrated use of antimicrobial stewardship is needed to monitor appropriate antibiotic use.

Symptoms of CDI can complicate diagnosis of COVID-19 because both conditions can have similar manifestations; in a study of 206 COVID-19 patients, $19.4 \%$ had diarrhea as the first symptom onset (10). Of the 2 patients who had CDI diagnosed at admission, 1 patient solely had gastrointestinal symptoms, which possibly led to delayed diagnosis of COVID-19. Both COVID-19 and CDI should be considered when evaluating patients with diarrhea during the COVID-19 pandemic. Distinguishing between actual CDI versus colonization also is vital; 1 patient in our cohort was colonized. A limitation of this study is the small number of cases. However, in the face of the COVID-19 pandemic and the extensive use of antibiotics, clinicians should remain aware of possible CDI and SARS-CoV-2 co-infection.

G.T. is a consultant to Melinta, Crestone, Ferring, AirMmax, and Shionogi. Other authors in the manuscript have no relevant conflict of interest or financial disclosure. No funding was needed for this manuscript.

\section{About the Author}

Dr. Sandhu is an infectious diseases-epidemiology fellow at Detroit Medical Center, Wayne State University School of Medicine. Her current research interest is in multidrugresistant hospital-acquired infections.

\section{References}

1. US Centers for Disease Control and Prevention. Interim clinical guidance for management of patients with confirmed coronavirus disease (COVID-19) [cited 2020 May 1]. https:/ / www.cdc.gov/coronavirus/2019-ncov/hcp/ clinical-guidance-management

2. Guan WJ, Ni ZY, Hu Y, Liang WH, Ou CQ, He JX, et al.; China Medical Treatment Expert Group for Covid-19. Clinical characteristics of coronavirus disease 2019 in China. N Engl J Med. 2020;382:1708-20. https:/ / doi.org/10.1056/ NEJMoa2002032

3. Chen N, Zhou M, Dong X, Qu J, Gong F, Han Y, et al. Epidemiological and clinical characteristics of 99 cases of 2019 novel coronavirus pneumonia in Wuhan, China: a descriptive study. Lancet. 2020;395:507-13. https:/ / doi.org/ 10.1016/S0140-6736(20)30211-7

4. Wang D, Hu B, Hu C, Zhu F, Liu X, Zhang J, et al. Clinical characteristics of 138 hospitalized patients with 2019 novel coronavirus-infected pneumonia in Wuhan, China. JAMA. 2020;323:1061-9. https://doi.org/10.1001/jama.2020.1585

5. Yang X, Yu Y, Xu J, Shu H, Xia J, Liu H, et al. Clinical course and outcomes of critically ill patients with SARS-CoV-2 pneumonia in Wuhan, China: a single-centered, retrospective, observational study. Lancet Respir Med. 2020;8:475-81. https:/ / doi.org/10.1016/S2213-2600 (20)30079-5

6. Chen T, Wu D, Chen H, Yan W, Yang D, Chen G, et al. Clinical characteristics of 113 deceased patients with coronavirus disease 2019: retrospective study. BMJ. 2020;368:m1091. https:// doi.org/10.1136/bmj.m1091

7. Brown KA, Khanafer N, Daneman N, Fisman DN. Meta-analysis of antibiotics and the risk of communityassociated Clostridium difficile infection. Antimicrob Agents Chemother. 2013;57:2326-32. https://doi.org/10.1128/ AAC.02176-12

8. Guh AY, Mu Y, Winston LG, Johnston H, Olson D, Farley MM, et al.; Emerging Infections Program Clostridioides difficile Infection Working Group. Trends in US burden of Clostridium difficile infection and outcomes. N Engl J Med. 2020;382:1320-30. https://doi.org/10.1056/NEJMoa1910215 
9. Biedron C, Chopra T. Issues surrounding antibiotic use in older adults. Curr Transl Geriatr Exp Gerontol Rep. 2013;2:151-8. https://doi.org/10.1007/s13670-013-0050-9

10. Han C, Duan C, Zhang S, Spiegel B, Shi H, Wang W, et al. Digestive symptoms in COVID-19 patients with mild disease severity: clinical presentation, stool viral RNA testing, and outcomes. Am J Gastroenterol. 2020 Apr 15 [Epub ahead of print]. https://doi.org/10.14309/ajg.0000000000000664

Address for correspondence: Teena Chopra, Wayne State University, Detroit Medical Center, 3990 John Rd, Detroit, MI 48201, USA; email: tchopra@med.wayne.edu

\section{SARS-CoV-2 RNA Detection on Disposable Wooden Chopsticks, Hong Kong}

\author{
Grace Lui, Christopher K.C. Lai, Zigui Chen, \\ Sylvia L.Y. Tong, Wendy C.S. Ho, Apple C.M. Yeung, \\ Siaw S. Boon, Rita W.Y. Ng, Paul K.S. Chan
}

Author affiliation: The Chinese University of Hong Kong Faculty of Medicine, Hong Kong, China

\section{DOI: https://doi.org/10.3201/eid2609.202135}

We detected severe acute respiratory syndrome coronavirus 2 (SARS-CoV-2) RNA on disposable wooden chopsticks used by 5 consecutive asymptomatic and postsymptomatic patients admitted for isolation and care at our hospital. Although we did not assess virus viability, our findings may suggest potential for transmission through shared eating utensils.

Tn late 2019, severe acute respiratory syndrome coronavirus 2 (SARS-CoV-2) emerged in China (1), spreading primarily through droplets and contact with respiratory secretions or fecal materials $(2,3)$. It has been shown that SARS-CoV-2 remains viable on plastic and stainless steel for 72 hours (4), and SARS-CoV on wood for 60 hours (5). Chopsticks have been essential eating utensils for $>3$ millennia, particularly in Asia, and are made mainly of wood and plastic; metal chopsticks are found in some countries, such as South Korea. Personal chopsticks are often used to pick food from communal dishes. We investigated whether chopsticks could be a potential vehicle of transmission for SARS-CoV-2.

We recruited 5 consecutive patients admitted for isolation and care at our hospital: 1 patient who was asymptomatic, 2 whose symptoms had subsided, 1 with moderate coronavirus disease (COVID-19) caused by SARS-CoV-2 infection, and 1 with severe COVID-19. Before mealtimes, each patient was given a pair of wooden chopsticks packed in a sealed plastic bag. These chopsticks are widely available in Hong Kong, including in canteens of public hospitals. They are made of plain wood, not bamboo, and not painted with color or lacquer. After mealtimes, we collected the used chopsticks. We dipped the tips of the chopsticks in $1 \mathrm{~mL}$ of phosphate-buffered normal saline and shook them for $30 \mathrm{sec}$ to release saliva and oral fluid. We detected SARS-CoV-2 RNA by quantitative reverse transcription PCR (6). We collected serial sputum samples and nasopharyngeal and throat swabs to document respiratory shedding and for comparison of viral RNA concentrations among specimen types. The Joint Chinese University of Hong Kong - New Territories East Cluster Research Ethics Committee approved this study.

Patient A, 47-year-old woman, was a close contact of a confirmed case-patient. Her diagnosis was based on a surveillance throat sample collected during quarantine. She was admitted to the hospital for isolation and appeared asymptomatic throughout her stay. A pair of chopsticks collected 2 days after admission (12 days after her last exposure) was positive for SARS-CoV-2 RNA (Figure). Two respiratory samples collected after admission were also positive. High-resolution computed tomography (HRCT) of her lungs revealed small consolidations and groundglass opacities in both lower lobes, left upper lobe, and right middle lobe.

Patient B, a 22-year-old woman, had a runny nose, headache, and fever develop on the day she returned from Europe. Her symptoms subsided after admission. Two chopsticks collected 1-2 days after symptoms had subsided were positive for SARSCoV-2 RNA (Figure). Viral RNA was detected from respiratory specimens until 8 days after symptoms had subsided. HRCT revealed small patchy groundglass opacity in the anterior segment of the left upper lobe of the lungs.

Patient C, a 67-year-old man with hypertension and minor coronary artery disease, had fever, cough with whitish sputum, and loose bowel movements develop 2 days after returning from Europe. Chopsticks collected 5 and 7 days after illness onset were positive for SARS-CoV-2 RNA (Figure). All respiratory 